\title{
Long Range Forward-Backward Correlations and the Color Glass Condensate
}

\author{
Néstor Armesto ${ }^{1}$, Larry McLerran ${ }^{2}$ and Carlos Pajares ${ }^{1}$ \\ 1 Departamento de Física de Partículas and IGFAE, \\ Universidade de Santiago de Compostela, \\ 15782 Santiago de Compostela, Spain \\ 2 Nuclear Theory Group and Riken Brookhaven Research Center, \\ Brookhaven National Laboratory Upton, NY 11793
}

July 27, 2021

\begin{abstract}
We discuss forward-backward correlations in the multiplicity of produced particles in heavy ion collisions. We find the Color Glass Condensate generates distinctive predictions for the long range component of this correlation. In particular, we predict the growth of the long range correlation with the centrality of the collision. We argue that the correlation for baryons is less strong than that for mesons.
\end{abstract}

\section{Introduction}

The Color Glass Condensate provides a phenomenologically successful description of high energy hadronic processes [1]-[11]. The basis of the description for the infinite momentum hadronic wavefunction is to decompose the wavefunction into pieces associated with the fast moving constituents, and a piece associated with the slower moving components [9]. The fast moving components act as sources for the slow moving components. The slow moving 
components are treated as classical fields. Because the density of the gluons in the hadronic wavefunction is large, the coupling constant is weak. The field strengths are large, nevertheless, $A \sim 1 / g$, and although one can use weak coupling techniques, the problem is essentially non-perturbative due to the strong field.

The physical picture of the soft component of the hadronic wavefunction can be easily understood in terms of the phase space distribution

$$
\eta=\frac{d N}{d y d^{2} p_{T} d^{2} r_{T}} .
$$

For $p_{T}<Q_{\text {sat }}$, where $Q_{\text {sat }}$ is the saturation momentum, $\eta \sim 1 / \alpha_{s}$. This means that the quantum mechanical gluon states are multiply occupied, and saturate at $1 / \alpha_{s}$. At this phase space density, repulsive interactions become large. As one adds more gluons to the system, these components of the hadronic wavefunction remain fixed, since the repulsive interaction energy makes this unfavorable relative to adding in a gluon in a less occupied state at higher transverse momentum. Above the saturation momentum, the phase space density becomes small. When one adds more gluons to the system, they typically have momenta above and near the saturation momentum. This increases the saturation momentum. The renormalization group equations for the Color Glass Condensate predict that the saturation momentum never saturates, that is, it grows with decreasing $x$ of the gluons in the wavefunction [12]-[15]. One is perpetually adding in more gluons at an ever increasing saturation momentum.

When applied to hadron-hadron collisions, one imagines the collision of two sheets of colored glass [16]-[23]. Initially, the CGC fields are transversely polarized color electric and magnetic fields. At a very short time after the sheets collide, $t \sim e^{-\kappa / \alpha_{s}}$, the fields become longitudinal electric and magnetic fields in the central region between the colliding nuclei, and the transverse field in this region vanishes. The original transversely polarized fields are associated with the fast moving components of the nuclei and remain intact. This situation is shown in Fig. 1. The matter produced immediately after the collision is called the Glasma. It has properties distinct from the Color Glass Condensate and the Quark Gluon Plasma. It exist at times intermediate between the two, and has some properties in common with both the CGC and the QGP, hence the name. 


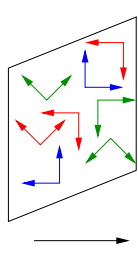

a
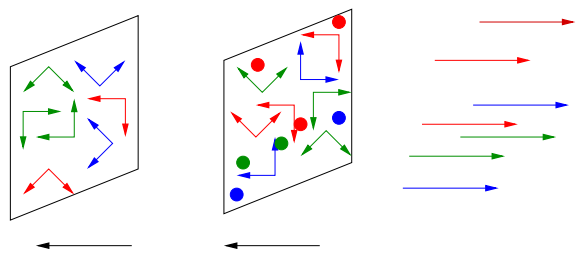

$\mathrm{b}$

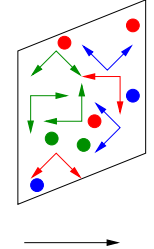

Figure 1: (a) Two sheets of colored glass approaching one another. (b) After the collision, an equal and opposite charge density of color electric and color magnetic charges are set up on each nucleus. This induces longitudinal color electric and magnetic fields in the region between the nuclei.

The origin of the longitudinal fields arises because as the sheets of colored glass pass through one another, the fast moving components have added to them a distribution of color electric and color magnetic charge. The charge density in the transverse plane of one nucleus is the negative of that in the other nucleus.

This picture of the fields has a restricted range of validity. For the effective action which describes these fields to be valid, we require that the field exist over a region of rapidity

$$
\Delta y \leq 1 / \alpha_{s}
$$

In a larger range of rapidity, quantum fluctuations become important, and the description in terms of classical fields breaks down.

It has also been argued that such long range correlations exist in the dual parton string picture of hadron collisions [24, 25]. Some time ago, it was also argued that the Color Glass Condensate has long range correlations in rapidity [26]. The Glasma provides a theoretical framework following directly from QCD which includes many features of the dual parton model approach, in particular the longitudinal rapidity structure.

In this paper, we explore these long range correlations. We use the formalism of forward-backward correlations. We argue that there is a long range 
component of fluctuations for the gluons,

$$
\left\langle\frac{d N_{F}^{g l}}{d y_{1}} \frac{d N_{B}^{g l}}{d y_{2}}\right\rangle \sim \frac{1}{\alpha_{s}} \frac{d N^{g l}}{d y},
$$

which is approximately rapidity independent. Here $F(B)$ are regions in the positive (negative) rapidity region, and $y$ is some rapidity intermediate between $y_{1}$ and $y_{2}$, oftentimes taken to be the center of mass rapidity. For quarks,

$$
\left\langle\frac{d N_{F}^{q}}{d y_{1}} \frac{d N_{B}^{q}}{d y_{2}}\right\rangle \sim \frac{d N^{q}}{d y}
$$

The essential difference between the two is that for the gluons the is a factor of $1 / \alpha_{s}$. This can be understood since the gluons are acting coherently. The decay process involves the coherent emission of $1 / \alpha_{s}$ gluons. In the language of clusters, the typical cluster size of produced by a fireball is $1 / \alpha_{s}$ particles. The quarks are fermions, and their typical cluster size is of order one, since there is no coherence for fermions.

To compute the forward-backward correlation function, we also need to know the short range component. We define ${ }^{1}$

$$
\sigma_{F B}=\frac{\left\langle N_{F} N_{B}\right\rangle-\left\langle N_{F}\right\rangle\left\langle N_{B}\right\rangle}{\left\langle N^{2}\right\rangle-\langle N\rangle^{2}},
$$

with $N$ the multiplicity in the forward or backward interval. We argue below that there is a short range component which falls exponentially in rapidity. It is of strength of order $\alpha_{s}^{2}$ relative to that of the long range component.

We will argue below that at short range, the correlation function is polluted by final state effects, and is not directly computed from the initial state distribution of gluons. If we look at the long range piece of $\sigma_{F B}$, say at rapidity separation of greater that one unit of rapidity, final state effects should not be so important.

We argue below that this long range component has the form

$$
\sigma_{F B}=\frac{1}{1+c \alpha_{s}^{2}} .
$$

\footnotetext{
${ }^{1}$ This quantity coincides with the $b$ usually defined through $\left\langle n_{F}\right\rangle\left(n_{B}\right)=a+b n_{B}[27]$.
} 
We do not perform a detailed computation of the constant $c$. It involves knowing the strength of the soft correlated emission. There is however a qualitative effect which we can understand about $c$ : If the contribution of baryons to the correlation function is large, then $c$ increases. This is because this contribution brings in no factor of $\alpha_{s}$. The contributions of baryons may be important of central collisions at large $p_{T}$.

Notice that as a function of increasing centrality, $\alpha_{s}$ decreases. The forward-backward correlation function should therefore increase for more central collisions.

\section{Computing the Forward-Backward Corre- lation}

Consider the collision of two nuclei in the center of mass frame. We treat the high momentum degrees as sources. There are sources for each nucleus. Let us consider some region of transverse extent $a$. The value of $a$ will be of the order of the inverse transverse momentum scale which we probe. For the total multiplicity fluctuations, this size is of the order $a \sim 1 / Q_{\text {sat }}$.

Now in the transverse region of size $a$, due to fluctuations, one of the nuclei will have a larger charge than the other. The situation becomes like that of $p A$ collisions where there is an asymmetry between target and projectile [28][29]. The total multiplicity is given by the number of gluons in the object with the smaller charge, evaluated at the saturation momentum scale of the larger charged object. This is because the larger charge object is effectively a black disk for the smaller charged object, and as such, during the scattering the smaller charged object materializes all of its gluons. Since the difference in charge between these objects should be small compared to the charge (so we can neglect the difference between the $Q_{\text {sat }}$ of projectile and target), for each region of size $a$, the total multiplicity is therefore of order

$$
\frac{d N}{d y} \sim \frac{1}{\alpha_{s}} a^{2} Q_{s a t}^{2} .
$$

Summing over all areas gives the familiar Kharzeev-Nardi formula for the total multiplicity [30],

$$
\frac{d N}{d y} \sim \frac{1}{\alpha_{s}} \pi R^{2} Q_{\text {sat }}^{2} .
$$




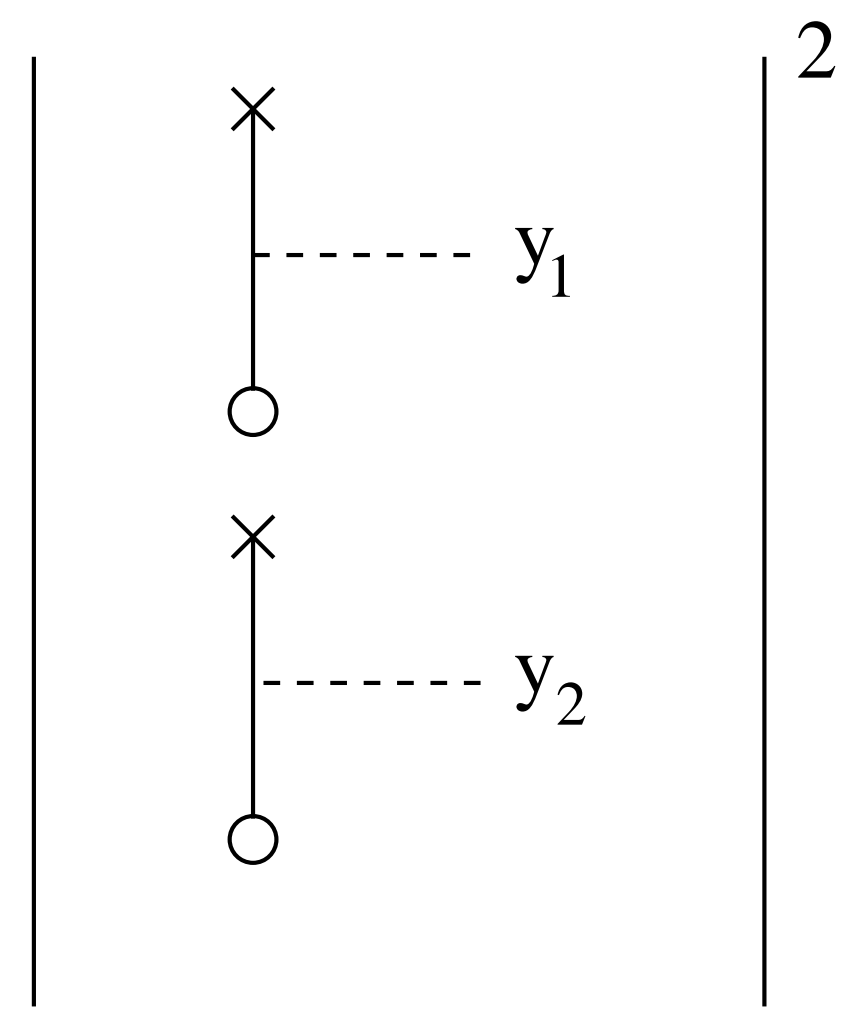

Figure 2: The leading order diagram which induce long range correlation in rapidity. The source of one nucleus is given by the $x$ and that of the other by the $o$. The produced gluon is denoted by the dotted line.

The fluctuations are given by the square of the diagram for the multiplicity. The leading order contribution from the classical fields comes from the diagram of Fig. 2. (This diagram at first sight appears to be uncorrelated, but becomes correlated after averaging over the sources associated with the nuclei. This averaging "ties together" the sources in the upper and lower part of the diagram.) There is a factor of $1 / \alpha_{s}^{2}$ which is due to the fact that this is the square of two classical processes. The correlation will only be non-zero if the same transverse area is probed. So the factor of $a^{4}$, upon summing over areas becomes of order $\pi R^{2} a^{2}$. There is also factor of $Q_{\text {sat }}^{4}$. For the total 
multiplicity squared, $a \sim 1 / Q_{\text {sat }}$, this gives

$$
\left\langle\left(\frac{d N}{d y}\right)^{2}\right\rangle \sim \frac{1}{\alpha_{s}^{2}} \pi R^{2} Q_{s a t}^{2} \sim \frac{1}{\alpha_{s}} \frac{d N}{d y} .
$$

The fluctuation at different rapidities is the same since the field which produces these particles is rapidity invariant:

$$
\left\langle\frac{d N}{d y_{1}} \frac{d N}{d y_{2}}\right\rangle=\left\langle\left(\frac{d N}{d y}\right)^{2}\right\rangle .
$$

Note the factor of $1 / \alpha_{s}$ in this result. This is because the $1 / \alpha_{s}$ gluons associated with the classical field behave coherently. Its origin is similar to that of the dependence on cluster size for fireball models of correlations. One needs to know the number of particles in each fireball decay. This number scales the square root in $N\left(\propto 1 / \alpha_{s}\right)$ fluctuation since one assumes each fireball is produced statistically but an extra factor of $N$ comes because each fireball decays into $N$ particles.

There is of course a correlated piece, which comes form the diagram of Fig. 3. Here one of the gluons is associated with the forward rapidity and the other with the backward rapidity. This diagram has two factors of $\alpha_{s}$, and should give a contribution to the total multiplicity fluctuations of order

$$
\left\langle\frac{d N_{c o r}}{d y_{1}} \frac{d N_{c o r}}{d y_{2}}\right\rangle \sim \alpha_{s} \pi R^{2} Q_{\text {sat }}^{4} \sim \frac{d N_{\text {cor }}}{d y} e^{-\kappa\left|y_{1}-y_{2}\right|} .
$$

Here $\kappa$ is a constant of order one. Note that $d N_{\text {cor }} / d y$ is of order $\alpha_{s}$ relative to that for the classical contribution. There is no interference with the leading order diagram of Fig. 2, since the average of an odd sources from the same nucleus must vanish. It should also be noted that outside of the context of perturbative QCD, there should be diagrams like Fig. 3 associated with the scattering of the hadronic degrees of freedom of the nuclei.

To understand the different factors of $\alpha_{s}$ between the diagrams of Fig 2 and Fig 3, we can think of the source as strong, and therefore of order $1 / \mathrm{g}$. Therefore each of the diagrams squared in Fig 2 is of order $1 / g^{2}$, making an overall contribution of order $1 / \alpha_{s}^{2}$. In Fig. 3, the diagram is overall zeroth order in powers of $g$. 


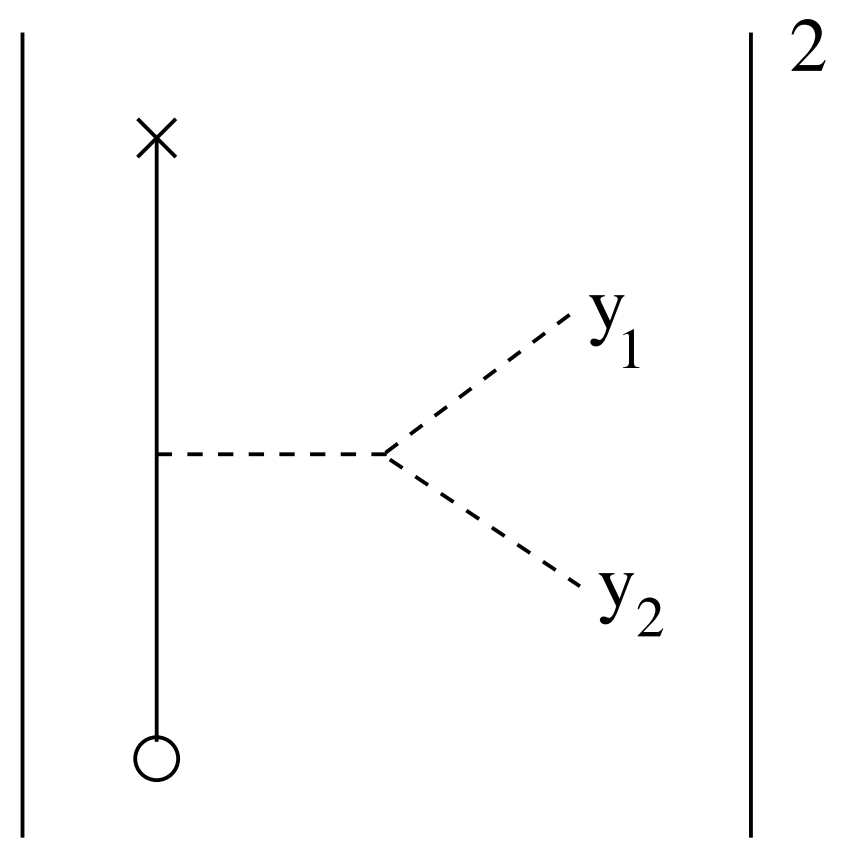

Figure 3: Here two gluon from the different sources scatter and produce two gluons in the forward and backward hemisphere.

For the long range correlation function, we see that the correlated piece is overall a factor of $\alpha_{s}^{2}$ smaller than the classical contribution. We therefore conclude that at large rapidities

$$
\sigma_{F B}=\frac{1}{1+c \alpha_{s}^{2}}
$$

where $\alpha_{s}$ is to be evaluated at the saturation momentum associated the centrality of the collision and we have considered two intervals $F, B$ symmetric around midrapidity. It clearly becomes more correlated as the centrality increases. It should also be noted that $c$ is an increasing function of the rapidity difference $\Delta y=\left|y_{1}-y_{2}\right|$. To see it, we define $\alpha_{s} \hat{c}=\frac{d N_{c o r}}{d y} / \frac{d N}{d y}$ to get

$$
c=\hat{c} \frac{1-e^{-\kappa \Delta y}}{1+\alpha_{s}^{2} \hat{c} e^{-\kappa \Delta y}},
$$


which clearly increases with increasing $\Delta y$.

We can repeat all the steps above for baryons. The only difference is that the baryon multiplicity is of order $\alpha$ relative to that of the gluons, and that in the fluctuation formula, there is no additional factor of $1 / \alpha_{s}$ associated with coherent emission. Thus the baryon correlation function should not grow with increasing centrality unlike of mesons which presumably ultimately arise from gluons.

In the most central collisions at RHIC, at high $p_{T}$, there is considerable contribution from baryons, and so measurements of correlations at high $p_{T}$ may not have such a large correlation for central collisions as would that for mesons.

In general, the correlations as a function of $p_{T}$ may be interesting. The low $p_{T}$ gluons are in a maximally occupied state. The repulsive interaction of the gluon in these states will not allow the addition of more gluons. Changing the charges on the nuclei therefore does not affect the multiplicity of low $p_{T}$ gluons, so there should not be large correlation. The dominant contribution to the total multiplicity fluctuations comes from gluons with $p_{T} \sim Q_{\text {sat }}$. For $p_{T}>Q_{\text {sat }}$, there should be considerable correlation. Of course the transverse momenta of gluons is strongly affected by final state interactions, and it is unclear how this effect could persist.

As a final comment, let us recall the result for forward-backward correlations in a different framework: string models which allow for some kind of collectivity [31]-[33] e.g. a non-thermal phase transition like percolation. The generic finding in these models is a reduction of the long range correlations with increasing collectivity. While this reduction increases with increasing centrality, it does not imply that the correlation itself reduces with increasing centrality for a given modeling of collectivity. Indeed, if $N_{s}$ is the number of independent particle sources and $n_{F}$ the number of particles in the forward hemisphere from each source, the same assumptions leading to Eq. (12) result here in

$$
\sigma_{F B}=\frac{1}{1+A}, \quad A=\frac{\left\langle N_{s}\right\rangle}{\left\langle N_{s}^{2}\right\rangle-\left\langle N_{s}\right\rangle^{2}} \frac{\left\langle n_{F}^{2}\right\rangle-\left\langle n_{F}\right\rangle^{2}}{\left\langle n_{F}\right\rangle^{2}} .
$$

$A$ is [34] a decreasing function with increasing centrality, so $\sigma_{F B}$ increases, as in the CGC, with increasing centrality. 


\section{Summary and Conclusions}

The strongest objection to this analysis is that we have used quarks and gluons as degrees of freedom. In reality, we measure mesons and baryons. It is standard lore that the meson multiplicity distribution directly reflects that of the gluons. Baryons, particularly baryon-antibaryon pairs may be subject to more drastic final state interactions. Baryons can be enhanced at intermediate $p_{T}$ in central collisions.

For long range correlations, we have not imagined a final state process which can affect the correlation in the total multiplicity. At short range, diffusion can have a strong effect. Imagine that after particles have been produced, some of the particles from the forward region diffuse to the backward region. This decreases the number of forward particles and increase those in the backward direction. This can induce a negative contribution to the correlation function. Certainly for rapidity differences of less than one unit, processes such as this will be important. That said, understanding such diffusion and comparison with correlation function measurements may probe such effects, and may be interesting in their own right.

Fluctuations in the correlation between centrality and impact parameter may give long range correlations. It is difficult to understand how the correlation could become stronger with more central collisions. Such correlations can be estimated by a variety of Monte Carlo event generators, and the strength of any resulting signal evaluated.

\section{Acknowledgments}

We thank the organizers of Quarks and Nuclear Physics 2006, where the discussions concerning the work presented above were initiated. We also thank Brijesh Srivastava for many enlightening conversations about how the forward-backward correlations are measured in the STAR experiment. Larry McLerran thanks Dima Kharzeev for insightful critical comments, and Rolf Scharenberg for useful insights. This paper was based on data presented at a seminar given at Brookhaven National Laboratory, July 27, 2006.[35]

This manuscript has been authorized under Contract No. DE-AC0298H10886 with the U. S. Department of Energy. NA is supported by Ministerio de Educación y Ciencia of Spain under a contract Ramón y Cajal. 
NA and CP acknowledge financial support by CICYT of Spain under project FPA2005-01963.

\section{References}

[1] L.V. Gribov, E.M. Levin and M.G. Ryskin, Phys. Rept. 100 (1983) 1.

[2] A.H. Mueller and J. Qiu, Nucl. Phys. B268 (1986) 427.

[3] I. Balitsky, Nucl. Phys. B463 (1996) 99; Phys. Lett. B518 (2001) 235.

[4] L. McLerran and R. Venugopalan, Phys. Rev. D49 (1994) 2233; ibid. 49 (1994) 3352; ibid. 50 (1994) 2225.

[5] Yu.V. Kovchegov, Phys. Rev. D54 (1996) 5463; ibid. D55 (1997) 5445.

[6] J. Jalilian-Marian, A. Kovner, L. McLerran and H. Weigert, Phys. Rev. D55 (1997) 5414.

[7] J. Jalilian-Marian, A. Kovner, A. Leonidov and H. Weigert, Nucl. Phys. B504 (1997) 415; Phys. Rev. D59 (1999) 014014.

[8] J. Jalilian-Marian, A. Kovner and H. Weigert, Phys. Rev. D59 (1999) 014015; A. Kovner, J. G. Milhano and H. Weigert, Phys. Rev. D62 (2000) 114005.

[9] E. Iancu, A. Leonidov and L. McLerran, Nucl. Phys. A692 (2001) 583; Phys. Lett. B510 (2001) 133; E. Ferreiro, E. Iancu, A. Leonidov and L. McLerran, Nucl. Phys. A703 (2002) 489.

[10] E. Iancu and L. McLerran, Phys. Lett. B510 (2001) 145.

[11] E. Iancu and R. Venugopalan, "The Color Glass Condensate and High Energy Scattering in QCD", arXiv:hep-ph/0303204, published in QuarkGluon Plasma 3, Eds. R.C. Hwa and X.-N. Wang, World Scientific, 2003.

[12] L.N. Lipatov, Sov. J. Nucl. Phys. 23 (1976) 338; E.A. Kuraev, L.N. Lipatov and V.S. Fadin, Sov. Phys. JETP 45 (1977) 199; Ya.Ya. Balitsky and L.N. Lipatov, Sov. J. Nucl. Phys. 28 (1978) 822. 
[13] E. Iancu, K. Itakura, and L. McLerran, Nucl. Phys. A708 (2002) 327

[14] A.H. Mueller and D.N. Triantafyllopoulos, Nucl. Phys. B640 (2002) 331

[15] D.N. Triantafyllopoulos, Nucl. Phys. B648 (2003) 293

[16] A. Kovner, L.D. McLerran and H. Weigert, Phys. Rev. D52 (1995) 6231.

[17] A. Kovner, L.D. McLerran and H. Weigert, Phys. Rev. D52 (1995) 3809.

[18] A. Krasnitz and R. Venugopalan, Nucl. Phys. B557 (1999) 237.

[19] A. Krasnitz and R. Venugopalan, Phys. Rev. Lett. 84 (2000) 4309.

[20] A. Krasnitz and R. Venugopalan, Phys. Rev. Lett. 86 (2001) 1717.

[21] A. Krasnitz, Y. Nara and R. Venugopalan, Phys. Rev. Lett. 87 (2001) 192302.

[22] T. Lappi, Phys. Rev. C67 (2003) 054903

[23] T. Lappi and L. McLerran, Nucl. Phys. A772, (2006) 200.

[24] A. Capella, U. Sukhatme, C. I. Tan and J. Tran Thanh Van, Phys. Rep. 236 (1994) 225.

[25] A.B. Kaidalov and K.A. Ter-Martirosyan, Sov. J. Nucl. Phys. 40 (1984) 135 .

[26] Y. Kovchegov, E. Levin and L. McLerran, Phys. Rev. C63 (2001) 024903.

[27] A. Capella and A. Krzywicki, Phys. Rev. D18 (1978) 4120.

[28] Y. Kovchegov and A.H. Mueller, Nucl. Phys. B529 (1998) 451.

[29] A. Dumitru and L. McLerran, Nucl. Phys. A700 (2002) 492.

[30] D. Kharzeev and M. Nardi, Phys. Lett. B507 (2001) 121.

[31] N.S. Amelin, N. Armesto, M.A. Braun, E.G. Ferreiro and C. Pajares, Phys. Rev. Lett. 73 (1994) 2813. 
[32] N. Armesto, M.A. Braun, E.G. Ferreiro and C. Pajares, Phys. Rev. Lett. 77 (1996) 3736.

[33] M.A. Braun, R.S. Kolevatov, C. Pajares and V.V. Vechernin, Eur. Phys. J. C32 (2004) 535.

[34] L. Cunqueiro, E.G. Ferreiro, F. del Moral and C. Pajares, Phys. Rev. C72 (2005) 024907.

[35] B. Srivastava, Seminar at Brookhaven National Laboratory, July 27, 2006, for the STAR collaboration.. 